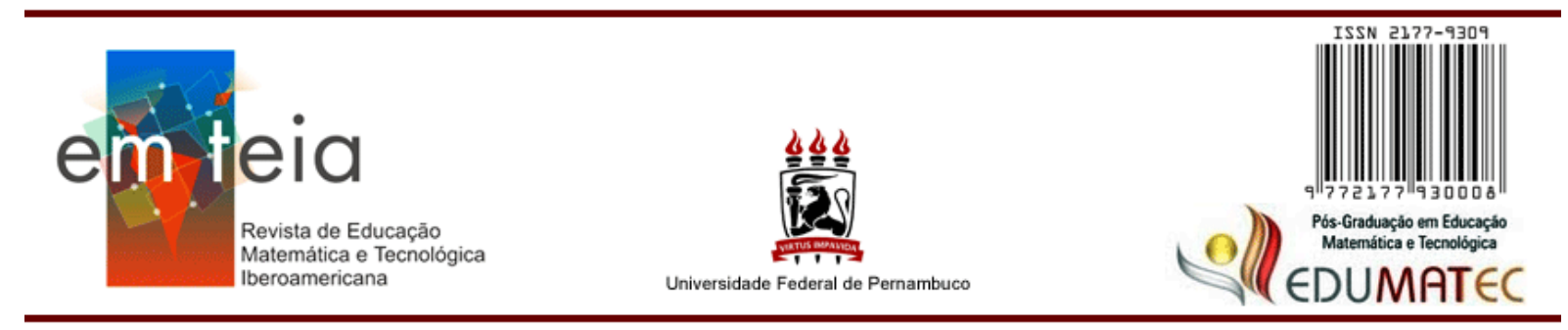

\title{
O ESTUDO DE EQUAÇÕES DIFERENCIAIS ATRAVÉS DA APLICAÇÃO DO PERFIL LOGARÍTMICO DO VENTO
}

\section{THE STUDY OF DIFFERENTIAL EQUATIONS THROUGH APPLICATION OF THE WIND LOGARITHM PROFILE}

Leandro Blass
leandroblass@unipampa.edu.br

André Felipe Huguenim andrehuguenim@hotmail.com

Valesca Brasil Irala valesca.irala@unipampa.edu.br

Vitória da Silva Farias

vitoriasfarias@hotmail.com

\section{Resumo}

Este artigo é resultado de uma estratégia didática desenvolvida na disciplina de Equações Diferenciais (ED) para cursos de exatas e nasceu de uma proposta de seminário avaliativo produzido por discentes de ED oriundos do curso de Engenharia de Energia. O objetivo da proposta do seminário é intercalar o conteúdo programático trabalhado em sala de aula com uma aplicação prática, preferencialmente voltada à formação profissional dos estudantes. $\mathrm{O}$ tema aqui escolhido é voltado à realização de análises do perfil logarítmico do vento, a partir do conhecimento das condições iniciais do problema em questão. O perfil logarítmico do vento é uma das muitas ferramentas existentes para estimar o perfil dos ventos (CAMELO et al., 2008; JERVELL, 2008). O estudo se justifica pela necessidade de os alunos das diversas engenharias perceberem como o conteúdo abordado em ED pode ser aplicado à sua área de formação. Para isso, foram trazidas neste artigo algumas estratégias para resolução e análise dos resultados a partir da abordagem dos discentes. Na resolução, adotou-se um passo a passo dos cálculos com diferentes métodos: separação de variáveis e exata. A solução do problema foi explorada de forma detalhada, a partir da abordagem feita em sala de aula, tais como: a solução analítica de uma Equação Diferencial Ordinária (EDO) de primeira ordem, a aplicação em um problema prático e a interpretação gráfica das soluções.

Palavras-Chave: Equações Diferenciais. Características do Vento. Aplicação Matemática. Interpretação gráfica. Engenharia de Energia.

\footnotetext{
Abstract

This article is the result of a didactic strategy developed in the subject of Differential Equations (DE) for exact courses and it comes from a proposal of an evaluative seminar produced by DE students from the Energy Engineering undergraduate course. The purpose of the seminar proposal is to merge
} 
the programmatic content created in the classroom with a practical application, preferably focused on the professional training of students. The theme chosen here is focused on the analysis of the logarithmic profile of wind from knowledge of the initial conditions of the problem in question. The logarithmic wind profile is one of the many tools available to estimate wind profile (CAMEL et al., 2008; JERVELL, 2008). This study is justified by the need for the students of various engineering fields to understand how the content addressed during the DE subject can be applied to their training area. For this reason, some strategies for solving and analyzing the results from the students' approach were brought into this article. In the resolution, a step by step calculation was adopted with different methods: variable separation and exact. Thus, the solution of the problem was explored in detail, considering some points worked in the classroom, such as: the analytical solution of a first order Ordinary Differential Equation (ODE), the application into a practical problem and graphical interpretation solutions.

Keywords: Differential Equations, Wind Characteristics, Mathematical Application, Graphical interpretation, Energy Engineering undergraduate course.

\section{Introdução}

Muitas pesquisas realizadas no cenário do Ensino Superior atualmente têm se preocupado em desenvolver estratégias didáticas (FERRARI; SÁENZ, 2018) que visam qualificar o processo de ensino-aprendizagem de Equações Diferenciais (ED), buscando contextualizar os saberes construídos nesse campo disciplinar e, também, contribuir na motivação e superação das dificuldades dos estudantes nessa disciplina, ofertada nos currículos de graduação de diversos cursos de exatas nas instituições de ensino superior brasileiras (DULLIUS; ARAÚJO; VEIT, 2011; DULLIUS, 2013; IGLIORI; ALMEIDA, 2017; OLIVEIRA; IGLIORI, 2013).

Como estratégia didática e avaliativa neste campo disciplinar, tem-se proposto aos discentes de ED de uma instituição pública localizada no sul do país o exercício de produzirem seminários (GONÇALVES, 2010; DOLZ et al., 2010), os quais são entendidos neste artigo como um gênero acadêmico que requer do estudante um conjunto sistemático e planejado de atividades (portanto, envolvem uma construção), o qual culmina em um produto, escrito e oral, de um elaborado e complexo processo de problematização dos tópicos abordados pela área em questão.

No presente artigo, expandimos e aperfeiçoamos um trabalho oriundo dessa experiência nascida em sala de aula, produzido por discentes do curso de Engenharia de Energia, a fim de contribuir para a disseminação de aplicações conectadas aos interesses formativos de diferentes estudantes de graduação e, também, motivá-los a valorizar a importância de áreas do conhecimento que não pertencem ao núcleo das disciplinas de conteúdos profissionalizantes e de conteúdos específicos, como é o caso de ED, que pertence 
ao chamado núcleo de conteúdos básicos (BRASIL, 2002; UNIVERSIDADE FEDERAL DO PAMPA, 2018).

A aplicação desenvolvida neste trabalho faz com que os estudantes de graduação, através das interpretações conduzidas por meio de representações gráficas, compreendam o conceito matemático mobilizado, representando a função resultante não apenas de forma algébrica. Nesse sentido, coincidimos com a reflexão do autor, que conclui que "ninguém promove a aprendizagem de conteúdos que não domina nem a constituição de significados que não possui ou a autonomia que não teve oportunidade de construir" (PIRES, 2002, p. 48).

Também, destacamos a abordagem utilizada da resolução de problemas, em que o problema é o ponto de partida das atividades matemáticas e o provocador do processo de construção de conhecimentos. Na próxima seção, apresentamos o aporte teórico utilizado neste estudo, focado primeiramente em um tópico sobre o ensino de ED e, em um segundo tópico, nos estudos sobre os perfis dos ventos. Na sequência, trazemos os resultados e as suas análises e discussões. Para ilustrar esses resultados, são expostos gráficos com diferentes abordagens de resolução e também as reflexões produzidas pelos alunos ao imergirem na temática.

\section{Referencial Teórico}

Para um melhor entendimento do referencial teórico, ele será dividido em duas partes: a) um panorama sobre a questão do ensino de ED; b) uma revisão a respeito dos estudos sobre os perfis dos ventos.

\section{Ensino de ED}

É de suma importância, para uma efetiva aprendizagem, que os alunos conectem o estudo das equações diferenciais com a prática de cada engenharia e, ao fazerem, que aprendam a lidar com os conceitos mobilizados de forma criativa e inovadora:

\footnotetext{
A sociedade atual, principalmente com a presença das tecnologias de informação e comunicação, tem colocado alunos e professores diante de demandas por novas formas de aprender e ensinar, formas que promovam a criatividade, a iniciativa e que sejam menos enfadonhas para os alunos (ROSSI; ALLEVATO, 2013, p. 2).
}

Por outro lado, muitos alunos, no decorrer de sua vida acadêmica, deparam-se com questionamentos a respeito de como os conteúdos propostos nas disciplinas iniciais de cálculo 
são passíveis de aplicação nos cursos de engenharia, ou, ainda, como poderão ser utilizados na vida profissional futura.

Comparando o contexto de ensino das Equações Diferenciais - EDs hoje em dia com o que se tinha na metade do século passado, percebemos que os tipos de alunos são outros, as necessidades e exigências do mercado de trabalho não são as mesmas, assim como as ferramentas disponíveis, mas a maioria das aulas continuam, em essência, sendo ministradas da mesma forma. Os currículos precisam ser repensados e os avanços tecnológicos considerados (DULLIUS; ARAÚJO; VEIT, 2011, p. 5).

Torna-se necessário, portanto, destacar o conceito de equação diferencial em virtude da importância para outras áreas do conhecimento, conforme pontuam Boyce e DiPrima (1999, p. IX):

[ ]... as equações mais simples correspondem a modelos físicos úteis, como por exemplo, o decaimento de substâncias radioativas, o comportamento de sistemas de massas e molas e o comportamento de circuitos elétricos. A partir dos modelos mais simples pode se obter modelos mais complexos.

Alguns autores, entre eles os aqui citados, trazem diversas aplicações em seus manuais didáticos voltados ao ensino dessa disciplina, os quais visam contribuir para que o aluno possa identificá-las com a área de seu interesse. Não é de hoje que se tem advogado em prol de que a visualização dos dados seja explorada a partir da construção e análise gráfica das equações que modelam o fenômeno estudado, através de recursos computacionais, contrapondo-se a uma visão mais tradicional do ensino dessa disciplina, já que

Estudos apontam que a metodologia dominante no contexto do ensino de equações diferenciais (EDs) está fortemente voltada para a resolução analítica, mas os recursos computacionais hoje disponíveis permitem ir além da mera aplicação de técnicas, podendo auxiliar os alunos na interpretação das equações diferenciais e suas soluções (DULLIUS, 2013, p. 1).

O modelo de resolução de problemas já é, há bastante tempo, uma alternativa para um ensino mais qualificado desse campo do conhecimento (POLYA, 1995) e se consolida como experiência didática no contexto investigado, por coadunar de forma coerente tanto com as demandas discentes quanto com a visão docente sobre a necessidade de tornar o ensino de ED menos desconectado dos demais eixos formativos dos diversos cursos para os quais é componente curricular ofertado, como é o caso do curso de Engenharia de Energia, foco deste artigo. Na próxima subseção, tal relação é explicitada, a partir do tema do perfil logarítmico do vento. 


\section{Estudos dos Perfis do Vento}

O presente trabalho trata sobre o tema do perfil logarítmico do vento, mais concretamente, o movimento realizado pelo ar perto da superfície terrestre, chamado vento de camada limite. Desde a antiguidade, os seres humanos utilizam a força dos ventos. Também, os primeiros navegantes dependiam dos ventos para conduzir suas viagens pelos oceanos. Os moinhos de vento o aproveitavam para mover pedras ou moer grãos. Nos dias de hoje, os moinhos de vento são utilizados para movimentar turbinas que geram energia elétrica. A literatura especializada traz alguns trabalhos em diferentes contextos (LUCAS, 2019).

Desde a década de 1990, a geração de fontes alternativas de energia tem se desenvolvido no Brasil, o que veio a ampliar, ao longo do tempo, a produção de pesquisas sobre energias limpas e renováveis (CAMELO et al., 2008) e, na década seguinte, na criação de diversos cursos de graduação em Engenharia de Energia, até então inexistentes no país. $\mathrm{O}$ estudo do perfil logarítmico do vento pode ser útil ao campo de estudos da Engenharia de Energia, como detalharemos na sequência.

O perfil logarítmico do vento apresenta uma análise muito eficiente e é utilizado em modelagens atmosféricas, com a finalidade de se obter um prognóstico do vento e do tempo em geral.

A aplicação da modelagem atmosférica no prognóstico não apenas do vento, mas do tempo em geral é muito importante, pois seu uso traz uma série de benefícios para as mais diversas atividades econômicas da sociedade: turismo; agricultura; aviação civil; transporte marítimo; planejamento urbano, entre outros (RAMOS; LYRA; JÚNIOR, 2013, p. 3).

Apenas para mencionar um outro campo de aplicação da análise do perfil logarítmico do vento, podemos citar a sua eficiência para que se obtenha sucesso em lançamentos e operações aeroespaciais:

Para o sucesso de qualquer lançamento ou operação aeroespacial, é de vital importância o conhecimento das condições meteorológicas da região. Dentre tais variáveis, podem ser ressaltados os ventos (de superfície e altitude) como possíveis agentes de influência e/ou modificação na trajetória dos veículos (GISLER; FISCH; CORREA, 2011, p. 1).

Castro et al. (2009) desenvolveram um método de avaliação da eficiência produtiva de sistemas de geração de energia eólica. Tais autores destacam a importância de estudos que avaliem, entre outros aspectos, situações de baixa velocidade do vento, variações climáticas, interrupções de energia elétrica e manutenções (preventivas e corretivas) imprevistas na rede, considerados como os principais fatores que elevam o custo operacional das fazendas eólicas 
ao longo dos anos. Nesse sentido, diferentes abordagens, como a aqui apresentada, podem ser úteis no entendimento de problemáticas que abordam tais aspectos. Na seção seguinte, apresentaremos o contexto de desenvolvimento da pesquisa.

\section{Metodologia}

Como já abordado na introdução deste artigo, a temática aqui efetuada nasceu em um seminário proposto como forma de avaliação de um componente curricular de Equações Diferenciais de uma universidade pública localizada no sul do país, ofertado no segundo semestre de 2018 para turmas multicurso (além do curso de Engenharia de Energia, as turmas dessa disciplina costumam comportar discentes de mais quatro cursos de engenharia e, também, discentes de cursos de licenciaturas da área de exatas). Nesse componente curricular, o docente deixa a critério dos alunos a escolha do tema, de acordo com sua área de conhecimento. Diante do problema estabelecido, foram usados diferentes métodos de resolução.

A avaliação do componente conta com acompanhamento de rubricas (BROOKHART, 2013) desenvolvidas para qualificar o processo da elaboração e avaliação do seminário proposto. Todas as orientações docentes são disponibilizadas no ambiente virtual de aprendizagem Moodle. A única exigência, do ponto de vista do ensino, é que cada equipe de discentes deve utilizar um ou mais métodos estudados em aula e, após, efetuar as devidas análises.

Os alunos realizam pesquisa bibliográfica, escolhem o tema e o problematizam. Após, selecionam os métodos matemáticos (quando é possível resolver por mais de um) para resolução da equação diferencial. Nesse caso, os métodos utilizados foram separação de variável e exata (BOYCE; DIPRIMA, 1999). Para análise dos resultados, foram usados gráficos desenvolvidos no Excel, explorando diferentes cenários (condições iniciais para o problema). Foi efetuada a análise da solução para os diferentes perfis do vento e desenvolvida uma situação de ordem prática, relacionada ao entorno geográfico mais próximo. Na seção seguinte, detalharemos os resultados obtidos.

\section{Resultados e Discussões}

Existem várias maneiras de se chegar a uma previsão de um perfil de vento logarítmico, como, por exemplo, a teoria do comprimento da mistura, que foi desenvolvida por Prandtl, em 1925, o qual “desenvolveu sua hipótese do comprimento de mistura e propôs 
um modelo algébrico de turbulência" (SOUZA et al., 2011, p. 1). É possível também utilizar a teoria da similaridade, que "permite prever o comportamento de um fenômeno real (protótipo), através das observações de um modelo similar” (ANGELES, 2003). Rogers, Mcgowan e Manwell (2009) utilizaram uma análise do tipo de comprimento de mistura, dada por Wortman, para chegar à equação do perfil logarítmico do vento. Admite-se, segundo a dedução desses autores, que perto da superfície da Terra a equação do momento é descrita por:

$\frac{\partial \mathrm{p}}{\partial \mathrm{x}}=\frac{\partial}{\partial \mathrm{z}} \tau_{\mathrm{xz}}$

onde:

p é a pressão;

$x$ é a coordenada horizontal;

z é a coordenada vertical;

$\tau_{x z}$ é a tensão de cisalhamento na direção x, cuja normal coincide com z. Nessa região, a pressão é independente de $\mathrm{z}$, logo:

$$
\tau_{\mathrm{xz}}=\tau_{0}+\mathrm{z} \frac{\partial \mathrm{p}}{\partial \mathrm{x}}
$$

onde:

$\tau_{0}$ é a tensão de cisalhamento na superfície.

Perto da superfície, a variação da pressão é pequena, então o termo $\partial \mathrm{p} / \partial \mathrm{x}$ pode ser considerado nulo. A partir do modelo empírico proposto por Prandtl, conhecido como a teoria do comprimento de mistura (SOUZA et al., 2011), permite que a tensão de cisalhamento possa ser expressa como em Rogers, Mcgowan e Manwell (2009, p. 45)

$$
\tau_{\mathrm{xz}}=\rho \mathrm{l}^{2}\left(\frac{\partial \mathrm{U}}{\partial z}\right)^{2}
$$

onde:

$\rho$ é a densidade do ar,

$\mathrm{U}$ a componente horizontal de velocidade,

1 o comprimento de mistura e depende de $\mathrm{z}$.

Pode-se notar que U é usado de forma a significar que os efeitos da turbulência foram calculados. Logo, combinação das Equações 2 e 3, fornecidas por Rogers, Mcgowan e Manwell (2009, p. 45) é: 


$$
\frac{\partial \mathrm{U}}{\partial \mathrm{z}}=\frac{1}{1} \sqrt{\frac{\tau_{0}}{\rho}}=\frac{\mathrm{u}^{*}}{1}
$$

onde:

$$
\sqrt{\frac{\tau_{0}}{\rho}}=\mathrm{u}^{*} \text { é definido como a velocidade de atrito. }
$$

Ao assumirmos uma superfície lisa $1=\mathrm{Kz}$, com $\mathrm{K}=0,4$ (constante de von Kármán), a Equação 4 pode ser integrada diretamente de $z_{0}$ a $z$, onde $z_{0}$ é o comprimento da rugosidade superficial, que caracteriza a rugosidade do solo terreno. Isso produz o que Rogers, Mcgowan e Manwell (2009, p. 45) apontam:

$$
\mathrm{U}(\mathrm{z})=\frac{\mathrm{u}^{*}}{\mathrm{k}} \ln \left(\frac{\mathrm{z}}{\mathrm{z}_{0}}\right)
$$

Essa equação é conhecida como o perfil logarítmico do vento. A integração é do limite inferior de $z_{0}$ em vez de 0 , porque as superfícies naturais nunca são uniformes e suaves. A Tabela 1 apresenta alguns comprimentos aproximados de rugosidade superficial para vários tipos de terreno.

Tabela 1 - Comprimentos aproximados de rugosidade superficial para vários tipos de terrenos

\begin{tabular}{c|c}
\hline Descrição do terreno & $\mathbf{z}_{\mathbf{0}}$ \\
\hline Gelo ou lama & 0.01 \\
\hline Mar calmo e aberto & 0.20 \\
\hline Mar agitado & 0.50 \\
\hline Neve na superfície & 3.00 \\
\hline Gramado & 8.00 \\
\hline Pastagem áspera & 10.00 \\
\hline Campo pousio & 30.00 \\
\hline Cultivo & 50.00 \\
\hline Poucas árvores & 100.00 \\
\hline Muitas árvores, sebes ou poucos edifícios & 250.00 \\
\hline Florestas e bosques & 500.00 \\
\hline Subúrbios & 1500.00 \\
\hline Centro de cidades com edifícios & 3000.00 \\
\hline
\end{tabular}

Fonte: Traduzida pelos autores a partir de Rogers, Mcgowan e Manwell (2009, p. 46). 
A seguir, é apresentado o passo a passo indicado a facilitar ao leitor a visualização do desenvolvimento da solução, através do método de separação de variáveis. Iremos resolver a Equação 4 (em que l= Kz e u* é a velocidade de atrito do vento). Obtém-se a Equação 5, que é o perfil logarítmico do vento através do método:

$$
\frac{\mathrm{dU}}{\mathrm{dz}}=\frac{1}{\mathrm{Kz}} \mathrm{u}^{*}
$$

Com a separação das variáveis, aplica-se a integral dos dois lados da igualdade (BOYCE; DIPRIMA, 1999):

$$
\begin{aligned}
\int \mathrm{d} U & =\int \frac{\mathrm{u}^{*}}{\mathrm{Kz}} \mathrm{dz} \\
\mathrm{U}(\mathrm{z}) & =\frac{\mathrm{u}^{*}}{\mathrm{~K}} \int \frac{1}{\mathrm{z}} \mathrm{dz}
\end{aligned}
$$

Usa-se da definição, na qual a integral de z é integrável de $\mathrm{z}$ a zo:

$$
\mathrm{U}(\mathrm{z})=\frac{\mathrm{u}^{*}}{\mathrm{~K}} \ln (\mathrm{z})_{\mathrm{z}_{0}}^{\mathrm{z}}
$$

Dessa forma, temos com resultado a função que representa o perfil logarítmico do vento:

$$
\mathrm{U}(\mathrm{z})=\frac{\mathrm{u}^{*}}{\mathrm{~K}} \ln \left(\frac{\mathrm{z}}{\mathrm{z}_{0}}\right)
$$

Aqui, demonstramos que a equação pode ser resolvida por outro método, com o qual se chega à mesma solução.

$$
\frac{\mathrm{dU}}{\mathrm{dz}}=\frac{1}{\mathrm{Kz}} \mathrm{u}^{*}
$$

O primeiro passo é escrever a equação na forma geral das exatas: $M(U, z) d u+$ $\mathrm{N}(\mathrm{U}, \mathrm{z}) \mathrm{dz}=0$.

$$
\mathrm{dU}-\frac{1}{\mathrm{Kz}} \mathrm{u}^{*} \mathrm{dz}=0
$$


O segundo passo é fazer o teste que diz se a EDO é exata: $M(U, z)=1$ e $\mathrm{N}(\mathrm{U}, \mathrm{z})=-\frac{1}{\mathrm{Kz}} \mathrm{u}^{*}$ (BOYCE; DIPRIMA, 1999, p. 45).

$$
\frac{d M}{d z}=\frac{d N}{d U} \Rightarrow\left\{\begin{array}{l}
\frac{d M}{d z}=0 \\
\frac{d N}{d U}=0
\end{array}\right.
$$

Logo, é possível ser resolvida pelo método de exatas. Dessa forma, utilizaremos do diferencial total (BOYCE; DIPRIMA, 1999, p. 46):

$$
\overbrace{\frac{\partial \mathrm{F}}{\partial \mathrm{U}}}^{\mathrm{M}(\mathrm{U}, \mathrm{z})} \mathrm{dU}+\overbrace{\frac{\partial \mathrm{F}}{\partial \mathrm{z}}}^{\mathrm{N}(\mathrm{U}, \mathrm{z})} \mathrm{dz}=0
$$

Escolhe-se um termo no Diferencial Total para iniciar os cálculos, aplicando a integral dos dois lados da igualdade:

$$
\int \frac{\partial F}{\partial U}=\int 1 d z \Rightarrow F(U, z)=U+g(z)
$$

O próximo passo é fazer a derivada da função $\mathrm{F}$ :

$$
\frac{\partial \mathrm{F}(\mathrm{U}, \mathrm{z})}{\partial \mathrm{z}}=+\mathrm{g}^{\prime}(\mathrm{z})=-\frac{1}{\mathrm{Kz}} \mathrm{u}^{*} \Rightarrow \mathrm{N}(\mathrm{U}, \mathrm{z})
$$

Aplica-se, assim, a integral dos dois lados:

$$
\int \mathrm{g}^{\prime}(\mathrm{z})=-\frac{\mathrm{u}^{*}}{\mathrm{~K}} \int \frac{1}{\mathrm{z}} \mathrm{dz} \Rightarrow \mathrm{g}(\mathrm{z})=-\frac{\mathrm{u}^{*}}{\mathrm{~K}} \ln (\mathrm{z})_{\mathrm{z}_{0}}^{\mathrm{z}}
$$

Após encontrar a função $\mathrm{g}(\mathrm{z})$, tem-se:

$$
\mathrm{U}(\mathrm{z})=\frac{\mathrm{u}^{*}}{\mathrm{~K}} \ln \left(\frac{\mathrm{z}}{\mathrm{z}_{0}}\right)
$$

Para a Figura 1, são consideradas a velocidade de atrito $\mathrm{u}^{*}=0.5 \mathrm{~m} / \mathrm{s}$, a constante de von Kármán $\mathrm{K}=0.4$, a altura $\mathrm{z}$ variando entre $1 \mathrm{~m}$ e $80 \mathrm{~m}$ e a rugosidade $\mathrm{z}_{0}=0.03 \mathrm{~m}$. 
Aplicamos esses valores na Equação 5 e obtivemos uma velocidade média do vento ideal de $10 \mathrm{~m} / \mathrm{s}$.

Figura 1 - Perfil Logarítmico do vento para velocidade média de $10 \mathrm{~m} / \mathrm{s}$

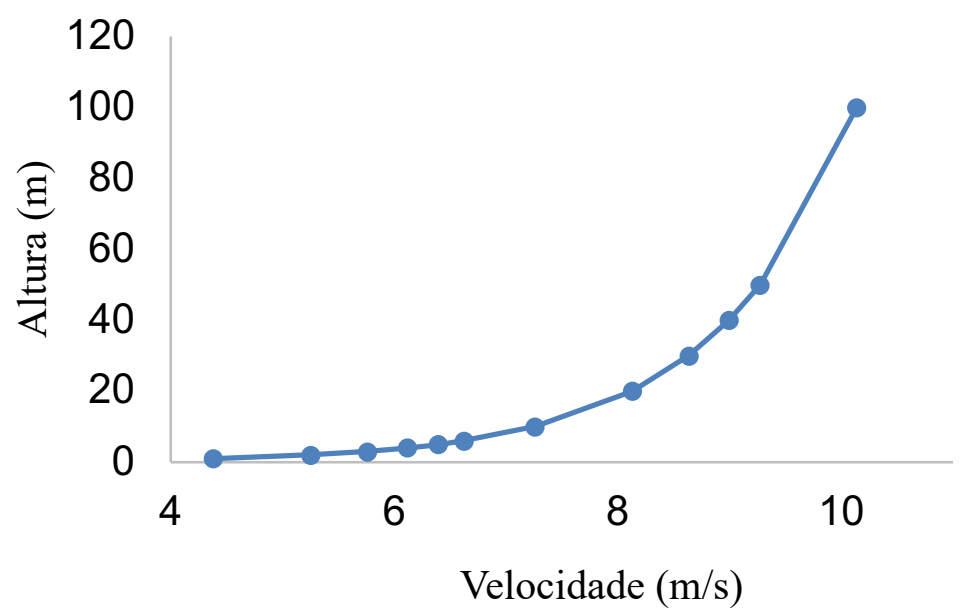

Fonte: Dados gerados pelos autores.

É possível observar que o perfil logarítmico do vento varia entre $4,38 \mathrm{~m} / \mathrm{s}$ e $9,86 \mathrm{~m} / \mathrm{s}$, aproximadamente, para alturas entre $1 \mathrm{~m}$ e $80 \mathrm{~m}$. A velocidade de atrito do vento varia entre $5 \%$ e $10 \%$, nesse caso. Para a Figura 1, considerou-se que a velocidade média fosse $10 \mathrm{~m} / \mathrm{s}$; logo, a velocidade de atrito do vento considerada foi $0,5 \mathrm{~m} / \mathrm{s}$. Esse perfil é considerado ótimo para geração de energia eólica, como apontam Rogers, Mcgowan e Manwell (2009).

Para a Figura 2, a condição inicial utilizada será $\mathrm{u}^{*}=0,425 \mathrm{~m} / \mathrm{s}, \mathrm{K}=0,4$, z variando entre $1 \mathrm{~m}$ e $80 \mathrm{~m}$ e $\mathrm{z}_{0}=0,03 \mathrm{~m}$. Para essa estimativa, a Figura 2 está apresentada a seguir:

Figura 2 - Perfil Logarítmico do vento para velocidade média de $8 \mathrm{~m} / \mathrm{s}$

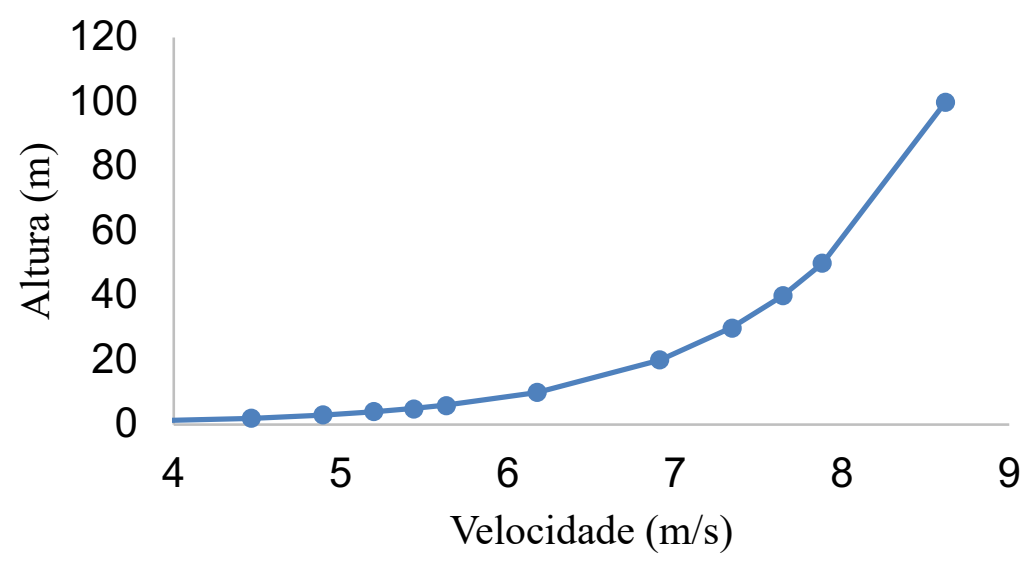

Fonte: Dados gerados pelos autores.

O perfil logarítmico do vento, nesse caso, inicia em 7,45m/s, aproximadamente, para $1 \mathrm{~m}$ e varia até $16,76 \mathrm{~m} / \mathrm{s}$, aproximadamente, para $80 \mathrm{~m}$. A velocidade média utilizada foi de 
$8,5 \mathrm{~m} / \mathrm{s}$, logo, a velocidade de atrito do vento foi de $0,85 \mathrm{~m} / \mathrm{s}$. Esse perfil é considerado mediano para geração de energia eólica, segundo Rogers, Mcgowan e Manwell (2009).

Para a Figura 3, a condição inicial utilizada foi $\mathrm{u}^{*}=0,375 \mathrm{~m} / \mathrm{s}, \mathrm{K}=0,4, \mathrm{z}$ variando entre $1 \mathrm{~m}$ e $80 \mathrm{~m}$ e $\mathrm{z}_{0}=0,03$ :

Figura 3 - Perfil Logarítmico do vento para velocidade média de 7,5 m/s

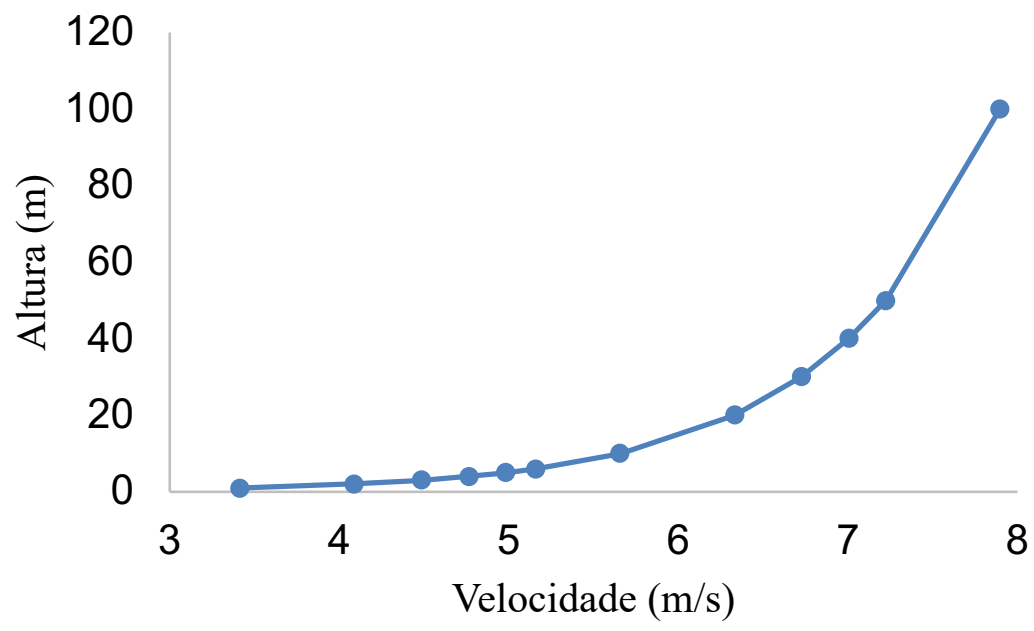

Fonte: Dados gerados pelos autores.

Esse perfil é considerado ruim para geração de energia eólica. Pode-se observar que se inicia em $6,57 \mathrm{~m} / \mathrm{s}$, aproximadamente, para $1 \mathrm{~m}$ e varia até $14,79 \mathrm{~m} / \mathrm{s}$, aproximadamente, para $80 \mathrm{~m}$.

Para as Figuras 1, 2 e 3, utilizaram-se os valores da velocidade média do vento. A Tabela 2 mostra essas classes de vento de acordo com a Comissão Eletrotécnica Internacional (IEC) e as variações de velocidades de vento que uma turbina deve ser projetada para suportar.

Tabela 2 - Classes de Vento IEC

Classe de vento IEC

\begin{tabular}{l|l|l|l}
\hline & $\mathbf{1}($ Vento alto) & $\mathbf{2}($ Vento médio) & $\mathbf{3}($ Vento fraco) \\
\hline Velocidade do vento de referência & $50 \mathrm{~m} / \mathrm{s}$ & $42,5 \mathrm{~m} / \mathrm{s}$ & $7,5 \mathrm{~m} / \mathrm{s}$ \\
\hline $\begin{array}{l}\text { Velocidade média anual do ano } \\
\text { (Máxima) }\end{array}$ & $10 \mathrm{~m} / \mathrm{s}$ & $8,5 \mathrm{~m} / \mathrm{s}$ & $7,5 \mathrm{~m} / \mathrm{s}$ \\
\hline Ritmo de retorno 50 anos & $70 \mathrm{~m} / \mathrm{s}$ & $59,5 \mathrm{~m} / \mathrm{s}$ & $52,5 \mathrm{~m} / \mathrm{s}$ \\
\hline $\mathbf{1}$ ano de retorno & $52,5 \mathrm{~m} / \mathrm{s}$ & $44,6 \mathrm{~m} / \mathrm{s}$ & $39,4 \mathrm{~m} / \mathrm{s}$ \\
\hline
\end{tabular}

Fonte: Adaptada pelos autores a partir de IEC (2019).

A Tabela 2 é fornecida pela IEC, que é uma organização de tecnologias elétricas, dentre outros assuntos (IEC, 2019). Através dessa tabela, é possível visualizar que a velocidade média anual do vento máxima varia entre $10 \mathrm{~m} / \mathrm{s}$ e $7,5 \mathrm{~m} / \mathrm{s}$. Com base nesses dados, foram desenvolvidos os cálculos do perfil logarítmico do vento, utilizando as 
velocidades médias de $10 \mathrm{~m} / \mathrm{s}, 8,5 \mathrm{~m} / \mathrm{s}$ e 7,5m/s. Tomando esses valores como condição inicial, foi possível visualizar o perfil logaritmo para ventos altos, médios e fracos.

A partir da Tabela 2, e com os resultados obtidos através das simulações anteriores, é possível chegar à conclusão de quais velocidades médias são recomendadas para a implantação de um parque eólico: a ideal é de $10 \mathrm{~m} / \mathrm{s}$ ou superior, a mediana de $8,5 \mathrm{~m} / \mathrm{s}$ e a ruim de $7,5 \mathrm{~m} / \mathrm{s}$ ou inferior.

Para avançar na contextualização da problemática estabelecida, buscou-se analisar o perfil do vento para a cidade de Bagé/RS, município sede da Universidade Federal do Pampa, onde o curso de Engenharia de Energia é ofertado. Segundo dados obtidos pela estação meteorológica de Bagé obtidos através do Instituto Nacional de Meteorologia (INMET), o perfil logaritmo do vento para os dias onde os horários que correspondem as maiores velocidade média do vento no intervalo de 05/06/2019 a 10/06/2019 é apresentado a seguir, na Figura 4. Para obter a figura que representa essa análise, a velocidade média do vento marcada no munícipio para esse horário foi $4,45848 \mathrm{~m} / \mathrm{s}$. Como mostrado na tabela 3 , para os cálculos que seguem, utilizaram-se $\mathrm{u}^{*}=0,25 \mathrm{~m} / \mathrm{s} ; \mathrm{K}=0,4 ; \mathrm{z}_{0}=0,03 \mathrm{e} \mathrm{z}$, variando entre $1 \mathrm{~m}$ e $80 \mathrm{~m}$.

Tabela 3 - Velocidade máxima do vento para cada dia

\begin{tabular}{|c|c|c|}
\hline Data & Hora & Velocidade do Vento $(\mathrm{m} / \mathrm{s})$ \\
\hline 05/06/2019 & $12: 00$ & 5,1444 \\
\hline $06 / 06 / 2019$ & $12: 00$ & 5,1444 \\
\hline $07 / 06 / 2019$ & $12: 00$ & 5,1444 \\
\hline $08 / 06 / 2019$ & 18:00 & 1,0288 \\
\hline $09 / 06 / 2019$ & 18:00 & 5,1444 \\
\hline $10 / 06 / 2019$ & $12: 00$ & 5,1444 \\
\hline
\end{tabular}

Para obter a Figura 4, que representa a análise do perfil do vento, foi calculada a velocidade média do vento segundo a Tabela 3, que resultou na média de $4,45848 \mathrm{~m} / \mathrm{s}$. As condições para a Equação (5) foram: $\mathrm{u}^{*}=0,25 \mathrm{~m} / \mathrm{s} ; \mathrm{K}=0,4 ; \mathrm{z}_{0}=0,03 \mathrm{e} \mathrm{z}$, variando entre $1 \mathrm{~m}$ e $80 \mathrm{~m}$.

Figura 4 - Perfil logarítmico do vento para cidade de Bagé (ano de 2019) 


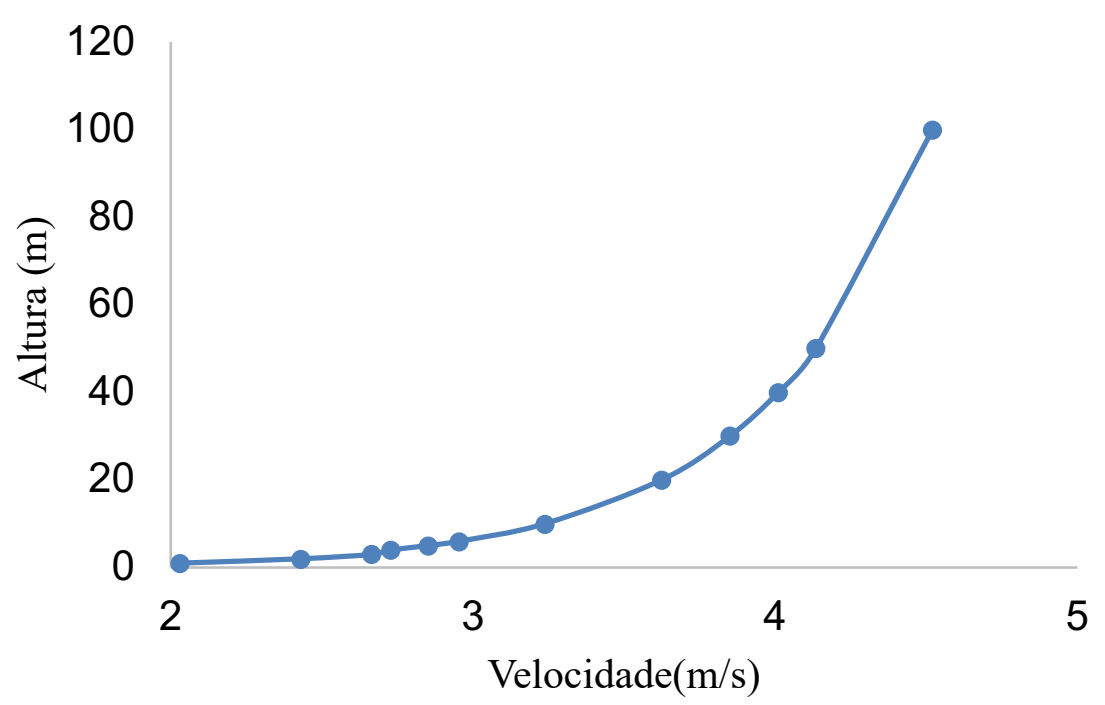

Fonte: Dados gerados pelos autores.

É possível perceber que uma velocidade média igual a 4,45848m/s é considerada baixa para a geração de energia, já que, segundo o IEC, abaixo de $7,5 \mathrm{~m} / \mathrm{s}$ são ventos fracos. Uma boa velocidade média para geração de energia está entre 8,5 e $10 \mathrm{~m} / \mathrm{s}$. Portanto, este perfil logaritmo do vento é considerado ruim e não seria viável a instalação de um parque eólico no município de Bagé sob essas condições.

Os dados encontrados neste trabalho dialogam com a constatação de Ramos, Krüger e Moraes (2013), os quais identificaram uma média de vento de 4,2 m/s durante a execução de sua pesquisa na mesma região, o que leva a considerar que vários estudos são necessários para definir, de forma categórica, a viabilidade de um parque eólico, considerando, inclusive, outros fatores aqui não apontados, bem como outros modelos de análise mais complexos (LUCAS, 2019).

Como aproximação inicial ao campo de investigação, do ponto de vista do processo de aprendizagem, no documento escrito produzido para o Seminário de Equações Diferenciais, os discentes relatam suas percepções sobre a validação da estratégia didática empregada:
“[...] tivemos a oportunidade de fixar melhor o conteúdo aprendido no decorrer do semestre, como também verificar a importância da disciplina e como ela se aplica ao curso de engenharia de energia e como fará parte da vida profissional, visto que, com o uso software excel e através das figuras, verificamos a importância e a realidade das EDOs no âmbito científico" (Trecho extraído do relato escrito dos estudantes).

Com esse relato dos discentes, evidenciamos que os objetivos propostos no âmbito da disciplina foram alcançados. Apresentou-se a equação do perfil logarítmico do vento através do método de separação de variáveis e exata e, através das análises realizadas da solução, foi 
possível visualizar o comportamento do perfil do vento, representado pela função resultante da solução da equação diferencial estudada, o que contribui para potencializar a aprendizagem e favorecer a implementação situada de melhorias no processo de ensino, considerando os interesses discentes e característica multicurso da disciplina.

\section{Considerações Finais}

O trabalho trouxe como resultado algumas observações dos docentes e discentes, que podem ser citadas:

a) $\mathrm{O}$ desenvolvimento de tais análises proporcionou aos estudantes uma visão mais contextualizada sobre o ensino de EDs, ao compreenderem que os métodos estudados podem ser transpostos à área de sua formação, o que, em geral, não lhes é tão evidente ao estudarem os componentes curriculares do núcleo de conteúdos básicos;

b) Com o desenvolvimento do trabalho, foi possível contemplar a possibilidade de interpretar e compreender o conceito de equação diferencial, a solução analítica, os métodos analíticos trabalhados em aula e a solução gráfica, assim, possibilitando a oportunidade da reflexão no contexto da disciplina, interligando-a ao Curso de Engenharia de Energia;

c) A aplicação desenvolvida dentro do contexto da vida profissional dos alunos é sem dúvida o ponto chave do trabalho, em função da contextualização da aprendizagem, pelo incentivo, na vida acadêmica, da resolução de um problema real da vida dos futuros engenheiros. Esse ponto vai ao encontro do registro dos discentes, em que eles citam a importância de se fazer um estudo aplicado, que efetivamente contribua para a sua profissão.

Por fim, é importante ressaltar que, do ponto de vista do ensino, são inúmeros os resultados obtidos junto aos estudantes de diversos cursos de engenharia, a fim de aproximálos, no interior de componentes curriculares como ED, de suas temáticas de interesse. As abordagens empregadas, bem como os seus ajustes e efeitos no desempenho discente, no contexto da instituição em questão, serão abordados em trabalhos futuros.

\section{Referências}


ANGELES, P. J. Estudo de tochas de plasma através da teoria da similaridade. 2003. 91f. Dissertação (Mestrado) - Universidade Estadual de Campinas, Instituto de Física Gleb Wataghin, Campinas, SP. Disponível em:

$<$ http://www.repositorio.unicamp.br/handle/REPOSIP/277402>. Acesso em: 3 ago. 2018.

BOYCE, W. E.; DIPRIMA, R. C. Equações Diferenciais Elementares e Problemas de Valores de Contorno. Tradução de Horácio Macedo. 6. ed. rev. Rio de Janeiro: LTC, 1999. 1040p.

BRASIL. Ministério da Educação, Conselho Nacional de Educação, Câmara de Educação Superior. Resolução CNE/CES No 11, de 11 de março de 2002 institui Diretrizes Curriculares Nacionais do Curso de Graduação em Engenharia. http://portal.mec.gov.br/cne/arquivos/pdf/CES112002.pdf. Acesso em: 13 abr. 2019.

BROOKHART, S. How to create and use rubrics for formative assessment and grading. Alexandria, Virginia (USA): ASCD, 2013. 174p.

CAMELO, H. N.; CARVALHO JUNIOR, P. C. M.; FILHO, J. B. V. L.; FILHO, J. B. P. A. Análise estatística da velocidade de vento do estado do Ceará. Rev. Tecnol. Fortaleza, v. 29, n. 2, p. 211-223, dez. 2008.

CASTRO, D. E.; PELLEGRINI, C. C.; LISBOA, A. H.; EDREIRA, S. M. Analysis of productive efficiency losses in wind energy conversion systems (WECS). In: 20th International Congress of Mechanical Enginering - COBEM 2009, Anais. Gramado (RS), Brasil, 15 a 20 de Novembro, 2009. Disponível em:

http://www.plusengenharia.com.br/files/artigos/artigo_id_6/Versao-final-CONEM-2009.pdf. Acesso em: 13 abr. 2019.

DOLZ, J. et al. A exposição oral. In: SCHNEUWLY, B.; DOLZ, J. (orgs.). Gêneros orais e escritos na escola. Campinas: Mercado de Letras, 2010. p. 183-212.

DULLIUS, M. M. Modelagem computacional para o Ensino de Equações Diferenciais Ordinárias em cursos de Engenharia. In: CONGRESO DE EDUCACIÓN MATEMÁTICA DE AMÉRICA CENTRAL Y DE EL CARIBE), 1., 2013, San Domingo. Anais...Disponível em: https://docplayer.com.br/8502395-Modelagem-computacional-para-o-ensino-deequacoes-diferenciais-ordinarias-em-cursos-de-engenharia.html. Acesso em: 13 jun. 2019.

DULLIUS, M. M.; ARAUJO, I. S.; VEIT, E. A. Ensino e Aprendizagem de Equações Diferenciais com Abordagem Gráfica, Numérica e Analítica: uma experiência em Cursos de Engenharia. Bolema. Boletim de Educação Matemática, v. 24, p. 17-42, 2011.

FERRARI, E. F.; SÁENZ, J. L. Didáctica práctica para enseñanza básica, media y superior. 3 ed. Montevideo: Magro, 2018. 514p. 
GISLER, C. A. F.; FISCH, G.; CORREA, C. S. Análise estatística do perfil de vento na camada limite superficial no centro de lançamento de Alcântara. J. Aerosp. Technol. Manag. [online], vol. 3, n. 2, p. 193-202, 2011.

GONÇALVES, A. V. Gênero seminário como objeto de ensino-aprendizagem: modelo didático. In: AZEVEDO, T.; PAVIANI, N. (orgs.). Universo acadêmico em gêneros discursivos. Caxias do Sul: EDUCS, 2010. p. 101-118.

IGLIORI, S. B.; ALMEIDA, M. V. Aplicações para o Ensino de Equações Diferenciais. Alexandria. R.Educ.Ci.Tec., v. 10, n. 1, p. 257-270, maio 2017.

INSTITUTO NACIONAL DE METEOROLOGIA (INMET). 2019. Disponível em: http://www.inmet.gov.br/portal/. Acesso em: 31 maio 2019.

INTERNATIONAL ELECTROTECHNICAL COMMISSION (IEC). 2019. Disponível em: https://www.iec.ch/. Acesso em: 24 abr. 2019.

JERVELL, J. T. Estudo da influência das características do vento no desempenho de aerogeradores. 2008. 87 f. Dissertação de Mestrado (Mestrado Integrado em Engenharia Mecânica), Faculdade de Engenharia da Universidade do Porto, 2008.

LUCAS, E. A. Pronóstico de Energía Eólica para Horizontes Temporales de Corto Plazo en Base a Modelo Numérico de Mesoescala y Redes Neuronales Artificiales. 2019. 168f. Dissertação (Programa de Posgrado en Ingeniería de la Energía), Universidad de la República, Montevidéu, 2019.

OLIVEIRA, E. A.; IGLIORI, S. B. Ensino e aprendizagem de equações diferenciais: um levantamento preliminar da produção científica. Em Teia - Revista de Educação

Matemática e Tecnológica Iberoamericana, Recife, v. 4, n. 2, p. 1-23, 2013.

PIRES, C. M. C. Reflexões sobre os cursos de Licenciatura em Matemática, tomando como referência as orientações propostas nas Diretrizes Curriculares Nacionais para a formação de professores da Educação Básica. Educação Matemática em Revista. Revista da Sociedade Brasileira de Educação Matemática. São Paulo, n. 11, p. 44-56, abr. 2002.

POLYA, G. A arte de resolver problemas. Trad. e adaptação Heitor Lisboa de Araujo. 2 reeimpr. Rio de Janeiro: Interciência, 1995. 196p.

RAMOS, D. N. S.; LYRA, R. F. F.; JÚNIOR, R. S. S. Previsão do vento utilizando o modelo atmosférico WRF para o estado de alagoas. Revista Brasileira de Meteorologia, v. 28, n. 2, p. 163-172, 2013.

RAMOS, L. F.; KRÜGER, C.; MORAES, M. R. Levantamento do potencial eólico da região da Campanha Meridional do Rio Grande do Sul com o modelo de mesoescala wrf e o modelo cfd windsim. DELOS Revista Desarrollo local sostenible, vol. 6 n.18, 1-12, 2013. 
ROGERS, A. L.; MCGOWAN, J. G.; MANWELL, J. F. Wind energy explained theory, design and application. Chichester: Wiley, 2009. 590p.

ROSSI, M.; ALLEVATO, N. Resolução de problemas no ensino de equações diferenciais. In: Congreso Iberoamericano de Educación Matemática. 7. 2013, Montevidéu. Anais...

Disponível em: http://www.cibem7.semur.edu.uy/7/actas/pdfs/772.pdf. Acesso em: 13 jun. 2019.

SOUZA, J. F. A.; OLIVEIRA, L. R.; AZEVEDO, J. L. L.; SOARES, I. D.; MATA, M. M. Uma revisão sobre a turbulência e sua modelagem. Revista Brasileira de Geofísica (Impresso), v. 29, p. 21-41, 2011.

UNIVERSIDADE FEDERAL DO PAMPA. Projeto pedagógico do curso de Engenharia de Energia. Bagé: UNIPAMPA, 2018. Disponível em:

http://dspace.unipampa.edu.br/bitstream/riu/95/6/PPC_Engenharia_de_Energia_Bagé.pdf. Acesso em: 13 abr. 2019. 